\title{
SUDDEN AND GRADUAL ALTERATION OF AMPLITUDE DURING THE COMPUTATION FOR FLOW AROUND A CYLINDER OSCILLATING IN TRANSVERSE OR IN-LINE DIRECTION
}

\author{
László Baranyi \\ Department of Fluid and Heat Engineering, \\ University of Miskolc, \\ H-3515 Miskolc-Egyetemváros, Hungary \\ E-mail: arambl@uni-miskolc.hu
}

\begin{abstract}
This study investigates the effect of altering oscillation amplitude on time-mean and root-mean-square values of force coefficients when plotted against amplitude of oscillation. The cylinder is placed in a uniform flow and is oscillated mechanically in transverse or in-line direction. The twodimensional numerical computations are carried out at $\mathrm{Re}=140$ and 160 , at $90 \%$ of the natural vortex shedding frequency. For in-line oscillation, jumps were found in the time-mean values of lift and torque. Both abrupt and gradual alteration of amplitude in the course of a computation had the effect of keeping the solution in one state curve, i.e., of conserving state, or inhibiting changes in vortex structure. Transverse oscillation displayed no jumps, and alteration of amplitude had no effect on the solution. Keywords: circular cylinder, in-line oscillation, lift, low Reynolds number flow, transverse oscillation
\end{abstract}

\section{INTRODUCTION}

Oscillating cylinders are of interest in situations such as off-shore structures and slender structures exposed to wind, but are also an area of fundamental research in the effort to clarify wake phenemona, in all its complexity. Transverse oscillation is most often studied, due to its relevance to real-life situations, while in-line oscillation is investigated by fewer researchers.

For a transversely oscillating cylinder, one landmark experimental study is that of Williamson and Roshko [1], in which they produced their well-known map of vortex synchronization regions at Reynolds number $\mathrm{Re}=392$, later adjusted for lower Re by Ponta and Aref [2]. Studies such as those of Blackburn and Henderson [3] and Kaiktsis et al. [4], at medium Reynolds numbers, found switches in the vortex structure with transverse oscillation. Gu et al. [5], $\mathrm{Lu}$ and Dalton [6], and Guilmineau and Queutey [7], in studies carried out for low Reynolds numbers $(\operatorname{Re}=185)$, also identified switches in vortex structure. Leontini et al. [8] determined the flow regimes for $\mathrm{Re}=100$ and 300 numerically, finding no influence of energy transfer sign switch on wake structures.

For cylinders oscillating in-line, one fundamental experimental study is Cetiner and Rockwell [9], investigating the flow over a wide frequency ratio and medium Reynolds number. Al-Mdallal et al. [10] carried out a study at the same wide frequency but at $\mathrm{Re}=200$, finding switches in vortex structure. Rodriguez and Mureithi [11] varied the amplitude of oscillation at medium Re to identify vortex wake modes.

While studies of both types of oscillation have identified switches in vortex structures, there seem to have been few studies, if any, that have investigated the influence of an alteration in oscillation amplitude. Baranyi [12] earlier found that altering the transverse amplitude of an orbiting cylinder led to the avoidance of vortex structure switches.

This study investigates a cylinder placed in a uniform stream and mechanically oscillated in either transverse or inline direction, varying the amplitude of oscillation within the lock-in domain, at $\mathrm{Re}=140$ and 160 and frequency ratio $f / \mathrm{St}_{0}$ of 0.9 . The primary purpose is to find whether switches in vortex structure occur under these conditions, and if so, whether this can be influenced by abruptly or gradually altering the amplitude of oscillation.

\section{NOMENCLATURE}

$a_{0} \quad$ cylinder acceleration, non-dimensionalized by $U^{2} / d$

$A_{x, y}$ amplitude of oscillation in $x$ or $y$ directions, respectively, non-dimensionalized by $d$

$C_{D} \quad$ drag coefficient, $2 F_{D} /\left(\rho U^{2} d\right)$

$C_{L} \quad$ lift coefficient, $2 F_{L} /\left(\rho U^{2} d\right)$

$C_{p b}$ base pressure coefficient 
$D$ dilation

$d \quad$ cylinder diameter $(\mathrm{m})$

$F_{D} \quad$ drag per unit length of cylinder $(\mathrm{N} / \mathrm{m})$

$F_{L} \quad$ lift per unit length of cylinder $(\mathrm{N} / \mathrm{m})$

$f$ oscillation frequency, non-dimensionalized by $U / d$

$f_{v} \quad$ vortex shedding frequency, non-dimensionalized by $U / d$

$p \quad$ pressure, non-dimensionalized by $\rho U^{2}$

Re Reynolds number, $U d / v$

St non-dimensional vortex shedding frequency

$T$ cycle period, non-dimensionalized by $d / U$

$t$ time, non-dimensionalized by $d / U$

$t_{q}$ torque coefficient, torque of shear stress on cylinder surface, non-dimensionalized by $\rho U^{2} d^{2}$

$U$ free stream velocity, velocity scale $(\mathrm{m} / \mathrm{s})$

$u, v$ velocities in $x, y$ directions, non-dimensionalized by $U$

$x, y \quad$ Cartesian co-ordinates, non-dimensionalized by $d$

$\Phi$ phase angle

$v \quad$ kinematic viscosity $\left(\mathrm{m}^{2} / \mathrm{s}\right)$

$\Theta$ polar angle characterizing initial condition

$\rho \quad$ fluid density $\left(\mathrm{kg} / \mathrm{m}^{3}\right)$

\section{Subscripts}

$D \quad$ drag

$\mathrm{fb} \quad$ fixed body

$L \quad$ lift

rms root-mean-square value

$v \quad$ vortex shedding

$x, y \quad$ components in $x$ and $y$ directions

0 for cylinder motion; for stationary cylinder at same $R e$

\section{GOVERNING EQUATIONS}

A non-inertial system fixed to the cylinder is used to compute two-dimensional low-Reynolds number unsteady flow around a circular cylinder placed in a uniform stream and forced to oscillate either in transverse or in-line direction. The non-dimensional Navier-Stokes equations for incompressible constant-property Newtonian fluid, the equation of continuity and the Poisson equation for pressure are as follows:

$$
\begin{gathered}
\frac{\partial u}{\partial t}+u \frac{\partial u}{\partial x}+v \frac{\partial u}{\partial y}=-\frac{\partial p}{\partial x}+\frac{1}{R e} \nabla^{2} u-a_{0 x} \\
\frac{\partial v}{\partial t}+u \frac{\partial v}{\partial x}+v \frac{\partial v}{\partial y}=-\frac{\partial p}{\partial y}+\frac{1}{R e} \nabla^{2} v-a_{0 y} \\
D=\frac{\partial u}{\partial x}+\frac{\partial v}{\partial y}=0 \\
\frac{\partial^{2} p}{\partial x^{2}}+\frac{\partial^{2} p}{\partial y^{2}}=2\left[\frac{\partial u}{\partial x} \frac{\partial v}{\partial y}-\frac{\partial u}{\partial y} \frac{\partial v}{\partial x}\right]-\frac{\partial D}{\partial t}
\end{gathered}
$$

In these equations, $u$ and $v$ are the $x$ and $y$ components of velocity, $t$ is time, $p$ is the pressure, $R e$ is the Reynolds number based on cylinder diameter, free stream velocity $U$, and kinematic viscosity $v$, and $D$ is the dilation. Although $D$ is theoretically equal to 0 by continuity, it is kept in equation (4) to avoid the accumulation of numerical errors.

On the cylinder surface, no-slip boundary condition is used for the velocity and a Neumann type boundary condition is used for the pressure. At the far region, potential flow is assumed.

Boundary-fitted coordinates are used to impose the boundary conditions accurately. Using unique, single-valued functions, the physical domain bounded by two concentric circles can be mapped into a rectangular computational domain where the spacing is equidistant in both directions (see Fig. 1). In the physical domain logarithmically spaced radial cells are used, providing a fine grid scale near the cylinder wall and a coarse grid in the far field. Using the mapping functions, not specified here, the governing equations and boundary conditions are transformed into the computational plane. The transformed equations are solved by finite difference method. Space derivatives are approximated by fourth order central differences, except for the convective terms for which a third order modified upwind scheme is used, [13]. The Poisson equation for pressure is solved by the successive overrelaxation (SOR) method. The Navier-Stokes equations are integrated explicitly and continuity is satisfied at every time step. For further details see [14] and [15].

The 2D code developed by the author has been extensively tested against experimental and computational results for a stationary cylinder ([16], [17], see [14], [15]) and computational results for cylinders oscillating in transverse or in in-line directions or following a circular path, including [6], [10] and [18], with good agreement being found, [15]. For this study the dimensionless time step was 0.0005 and the number of grid points $361 \times 236$. The computational domain is characterized by $R_{2} / R_{1}=60$ and the solution is grid independent.

\section{COMPUTATIONS}

Computations for a mechanically oscillated cylinder were carried out at two Reynolds numbers, $\mathrm{Re}=140$ and 160 . These are mid-range in the author's customary domain of investigations and the author has data for other cylinder flows at these Reynolds numbers. Both in-line (non-dimensional amplitude $\left.A_{x}\right)$ and transverse $\left(A_{y}\right)$ cylinder oscillation were investigated at varying amplitudes of oscillation. The nondimensional frequency of oscillation $f$ was set at $0.9 \mathrm{St}_{0}$, where $\mathrm{St}_{0}$ is the non-dimensional vortex shedding frequency, or Strouhal number, for a stationary cylinder at that Reynolds number. This selection of $f$ ensures synchronization or lock-in at moderate amplitude values of $A_{x}$ or $A_{y}$. In this study the flow was considered to be locked-in for both in-line and transverse cylinder oscillation when the vortex shedding frequency $f_{v}$ is equal to the frequency of cylinder oscillation $f$. For in-line oscillation, lock-in was found earlier in the vicinity of the natural vortex shedding frequency $f_{v 0}$, as well as the double of 
this frequency $[15,18]$. Here the first, narrower lock-in domain has been chosen.

To create a more manageable scope of investigation only locked-in cases were considered in this study. The cylinder was started at different positions, characterized by polar angle $\Theta$, since earlier investigations showed that this initial condition may have an effect on the solution, [15]. If not otherwise stated $\Theta=0^{\circ}$, meaning that the cylinder is started from its farthest downstream position ( $\Theta$ increases in the clockwise direction).

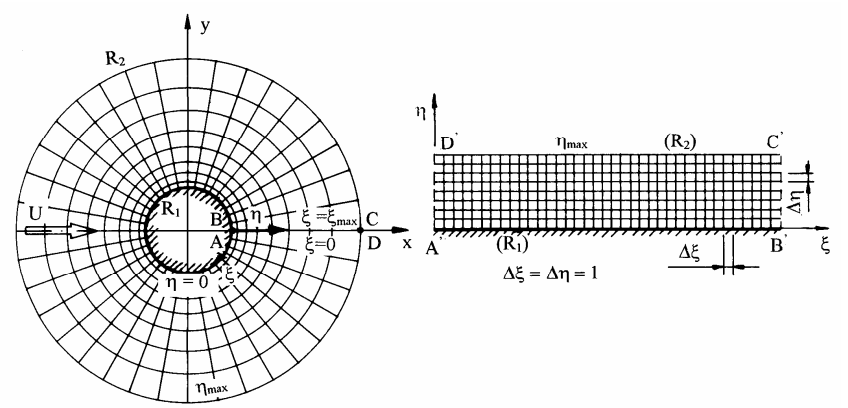

Figure 1. Physical and computational domains

Time-mean (TM) and root-mean-square (rms) values of lift $\left(C_{L}\right)$, drag $\left(C_{D}\right)$, base pressure $\left(C_{p b}\right)$ and torque coefficients were evaluated and plotted against the oscillation amplitude.

Throughout this paper the lift and drag coefficients used contain the inertial forces originated from the non-inertial system fixed to the accelerating cylinder. Coefficients obtained by removing the inertial forces are often termed 'fixed body' coefficients [6]. The relationship between the two sets of coefficients can be written as

$$
C_{L}=C_{L f b}+\frac{\pi}{2} a_{0 y}, \quad C_{D}=C_{D f b}+\frac{\pi}{2} a_{0 x}
$$

where subscript $f b$ refers to the fixed body (understood in an inertial system fixed to the stationary cylinder), [19], [20]. Since the inertial terms are $T$-periodic functions, their timemean values vanish, resulting in identical TM values for lift and drag in the inertial and non-inertial systems. Naturally the rms values of $C_{L}$ and $C_{D}$ will be somewhat different in the two systems.

Computations are performed at a fixed $\operatorname{Re}, f$ and $\Theta$ value with amplitude of oscillation as the independent variable. During one computation, the amplitude $\left(A_{y}\right.$ or $\left.A_{x}\right)$ is held constant. Later we will refer to this as the constant-amplitude solution.

In this study, we investigate the effect of altering the amplitude of the in-line or transverse oscillation either abruptly or gradually during the course of one computation, and compare this with the constant-amplitude (CA) solution. This was earlier investigated for a cylinder in orbital motion, [12].

\section{IN-LINE CYLINDER OSCILLATION}

The non-dimensional cylinder displacement of the center of the cylinder is described by

$$
x_{0}=A_{x} \cos (2 \pi f t+\Theta)
$$

where $A_{x}$ is the amplitude of in-line oscillation, $f$ is the frequency of cylinder oscillation, and $\Theta$ is the polar angle characterizing the initial condition for the cylinder motion. In equation (6) $t$ is the non-dimensional time.

This in-line cylinder oscillation can be considered as the limiting case of an orbiting cylinder when the amplitude of transverse oscillation $A_{y}$ tends to zero. For an orbiting cylinder, when the TM and rms values of force coefficients $\left(C_{L}, C_{D}, C_{p b}\right.$, $t_{q}$ ) are plotted against $A_{y}$ while $A_{x}$ is kept constant, two patterns emerge. The TM of $C_{L}$ and $t_{q}$ shows two envelope curves, or state curves, which are almost parallel with each other, and between which the solution jumps. State curves for the TM of $C_{D}$ and $C_{p b}$ and for all rms curves, on the other hand, intersect each other at $A_{y}=0$ [15]. For an orbiting cylinder jumps between the two state curves occur in all TM and rms curves. These jumps are associated with a sudden switch in the vortex structure [15]. There appear to be two attractors in this nonlinear system, and the solution is attracted to one or the other depending on the parameters and the initial condition.

The two patterns found for orbital motion are reflected in the results for in-line oscillation. In the first, for the TM of $C_{L}$ and $t_{q}$, double state curves are found. This is supported by the findings for orbital motion [15], where at the limiting case of $A_{y}=0$, two solutions can be found for the TM of lift and torque, i.e., two distinct points of the state curve exist. The second pattern, for the other six curves, appears in in-line oscillation as a single curve, which also fits in with the findings for orbital cylinder, where only one point is found at $A_{y}=0$.

\section{Constant-amplitude solutions}

For constant-amplitude (CA) solutions for in-line oscillation, two patterns are revealed when plotting the TM and rms values of force coefficients $\left(C_{L}, C_{D}, C_{p b}, t_{q}\right)$ are plotted against $A_{x}$. One is a double state curve, and the second appears to be a single curve.

The first pattern can be observed when looking at the TM of lift and torque coefficients. Similarly to earlier findings for the flow past an orbiting cylinder ([15] and [21]), abrupt jumps can be seen at certain $A_{x}$ values in these figures. Typical examples for the TM value of lift and torque coefficients are shown in Figs. 2 and 3 for $\mathrm{Re}=140, f=0.9 \mathrm{St}_{0}, \Theta=0^{\circ}, \mathrm{St}_{0}=0.1821$ ( $\mathrm{St}_{0}$ was taken from [22]).

It seems that there are two possible solutions and the actual solution jumps between these two state curves. It can be seen that the location and number of jumps are the same in these two figures. The shapes of the state curves (only portions of the curves are realized in the figures) are quite different for the TM of lift and torque. However, it can be seen that the state curves look as if they were mirror images of each other. This guess can 
be confirmed by investigating the effect of initial condition $\Theta$ on the solution. When repeating the computations for different $\Theta$ values the state curves remains unchanged but the starting point of computations (initial condition) leads to changes in the location and number of jumps. Using different $\Theta$ values, different parts of the state curves can be realized. Carrying out the computations for a few $\Theta$ values, the state curves can be reproduced nearly fully, as in the orbiting cylinder case ([15]).

Pre- and post-jump analysis showed radical changes through the jump. Time histories of lift are shown in Fig. 4. The pre-jump curve $\left(A_{x}=0.2887\right)$ and post-jump curve $\left(A_{x}=0.29\right)$ are almost mirror images of each other, meaning a $180^{\circ}$ phase shift between the two signals. A tiny change in the amplitude value is therefore capable of creating a drastic change in the curve. Vorticity contours are also almost mirror images, as in Fig. 5. This is also true for limit cycle curves $\left(x_{0}, C_{L}\right)$ (not shown here).

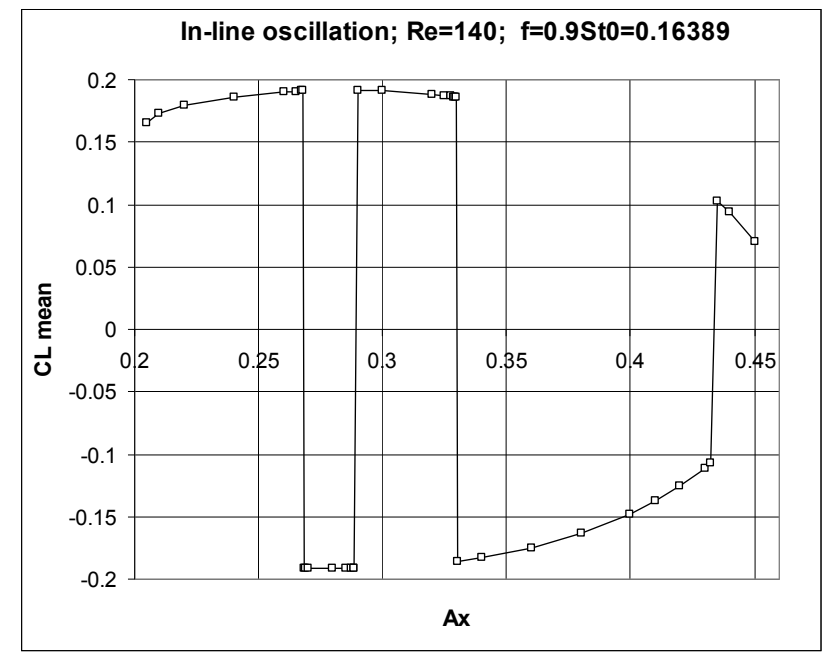

Figure 2. Time-mean value of lift versus amplitude $A_{x}$

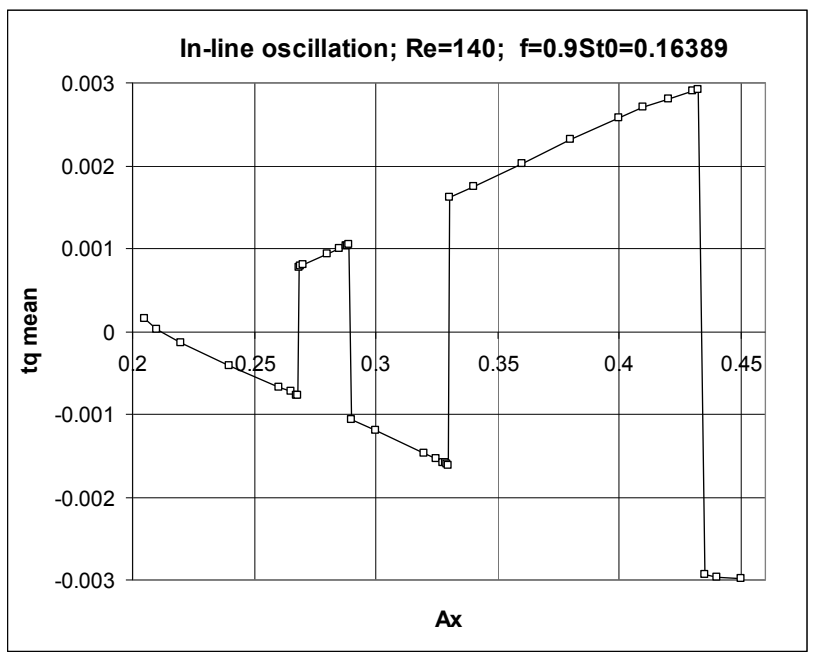

Figure 3. Time-mean value of torque versus amplitude $A_{x}$

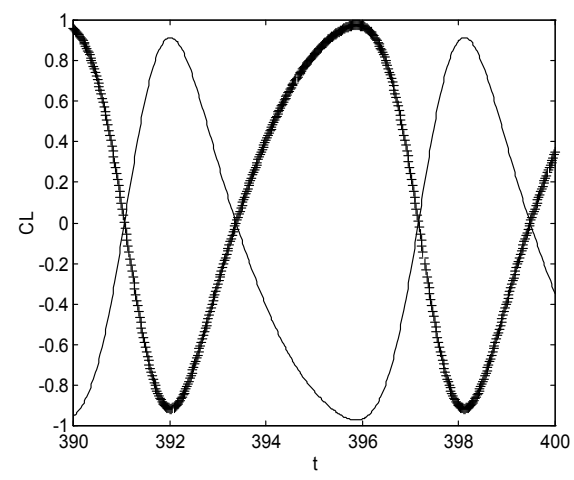

Figure 4. Time history of lift for $A_{x}=0.2887$ (thin line) and $A_{x}=0.29$ (thick line) $\left(\operatorname{Re}=140, A_{y}=0, f=0.16389, \Theta=0^{\circ}\right)$

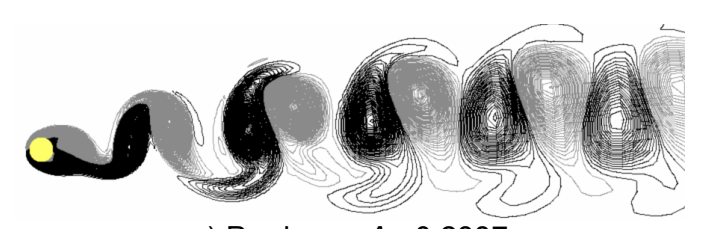

a) Pre-jump, $A_{x}=0.2887$

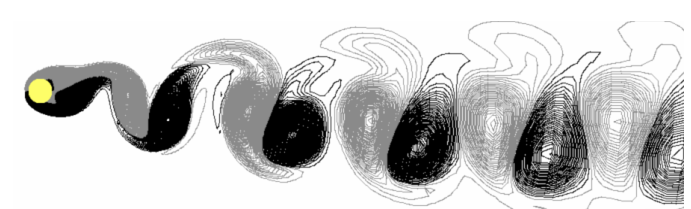

b) Post-jump, $A_{x}=0.29$

Figure 5. Vorticity contours before and after a jump for identical cylinder positions $\left(\operatorname{Re}=140, A_{y}=0, f=0.16389, \Theta=0^{\circ}\right)$

As examples of the second pattern, Figs. 6 and 7 show the dependence on the amplitude of oscillation $A_{x}$ for the TM of drag and the rms value of base pressure, respectively. As can be seen, there are no jumps in these curves, which is in accordance with the findings in [15], where at zero $A_{y}$ value there was just one solution for all rms values and for the TM values of drag and base pressure coefficients.

The TM values of $C_{L}$ and $t_{q}$ are distinguished from the rest because, for a stationary cylinder and for a cylinder oscillating transversely to the main stream, the lift and torque are the only values which depend on whether the vortex is shed from the upper or lower side of the cylinder, meaning that they are $T$ periodic. All rms values and the $C_{D}$ and $C_{p b}$ are independent of the location of vortex shedding, so are $T / 2$-periodic. It seems contradictory, however, that when the cylinder is oscillated in in-line direction or following an elliptical path [15] under lockin, any of the lift, drag, base pressure and torque coefficients has the same period, which is equal to the reciprocal value of cylinder oscillation frequency. It should be mentioned that when the effect of frequency ratio on the $\mathrm{TM}$ and $\mathrm{rms}$ of force 
coefficients was investigated in [23], jumps were also found in the TM value of lift and torque only.

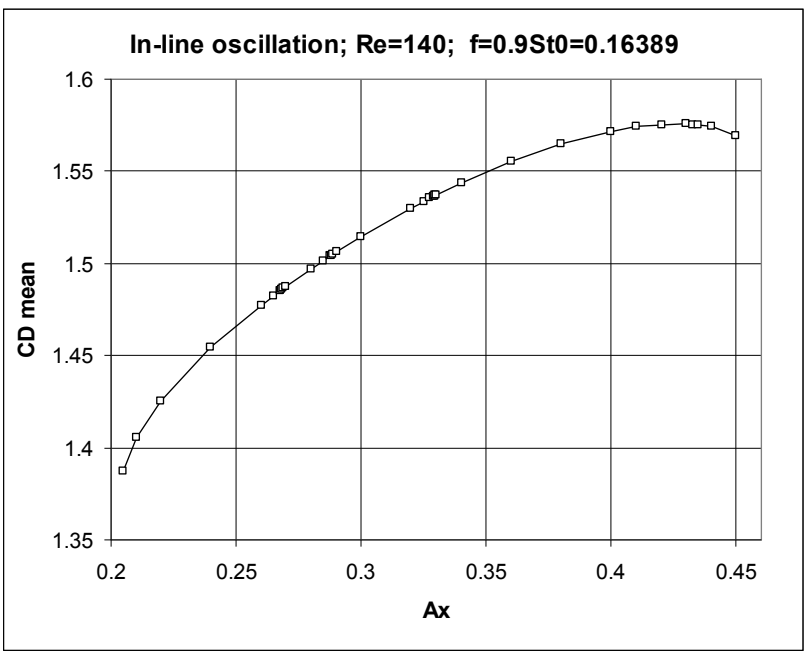

Figure 6. Time-mean value of drag versus amplitude $A_{x}$

Since no jumps occur in the curves for rms values, and TM values are not affected, it turns out that the selection of either the inertial or non-inertial system for the calculations of lift and drag yields no qualitative difference between the results in this case.

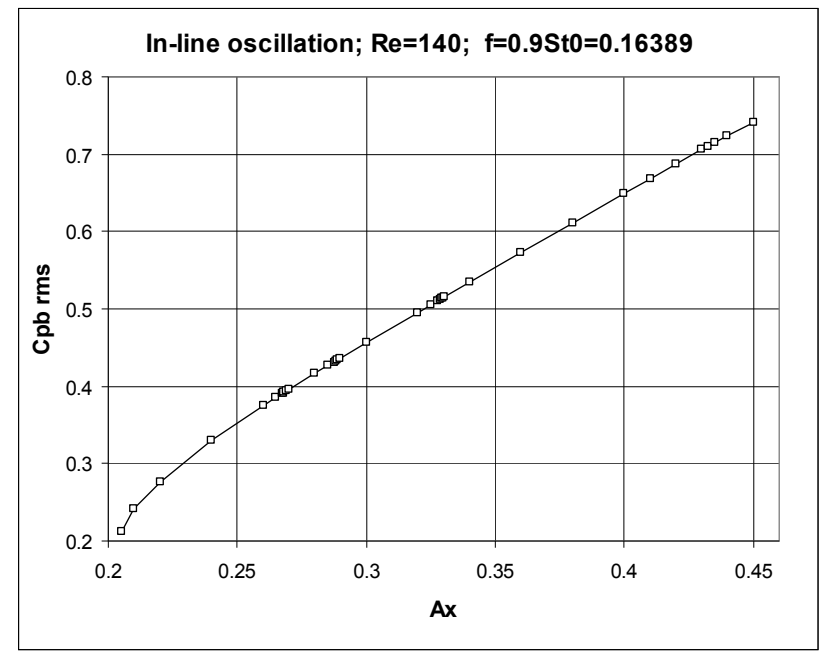

Figure 7. Rms value of base pressure versus amplitude $A_{x}$

Effect of initial condition Computations were repeated for $\mathrm{Re}=160, f=0.9 \mathrm{St}_{0}, \mathrm{St}_{0}=0.1882$ for $\Theta=0^{\circ}, 135^{\circ}$ and $270^{\circ}$. Results obtained are very similar in nature to those obtained for the case of $\mathrm{Re}=140$ for $\Theta=0^{\circ}$ shown above. Where two distinct state curves exist, they can be more fully realized by changing the initial condition, as shown in Fig. 8 for two different initial conditions. Where state curves coincide (not shown here), a change in initial condition produces no visible effect on TM or rms values of force coefficients.

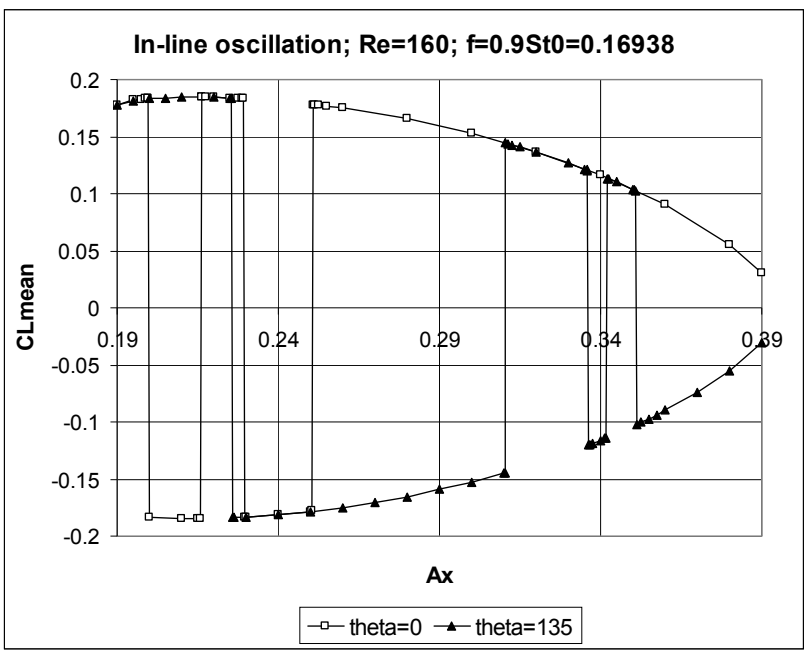

Figure 8. Time-mean value of lift for $\Theta=0^{\circ}$ and $135^{\circ}$ versus amplitude $A_{x}$

\section{Altered-amplitude solutions}

In the cases shown above, each point in the figure was obtained while keeping the parameters $\left(\mathrm{Re}, A_{x}, f / \mathrm{St}_{0}, \Theta\right)$ constant during the course of a single computation. To obtain the next point on the curve, amplitude was varied by changing $A_{x}$. The question arises as to what happens when the amplitude is changed during the course of the computation. This was investigated by altering $A_{x 1}$ to $A_{x 2}$ either abruptly at $t_{1}$ or gradually from $A_{x 1}$ to $A_{x 2}$ between $t_{1}$ and $t_{2}$, to either a larger or smaller value, and continuing the computation long enough to obtain a periodic solution. $A_{x 2}$ is chosen to cover the complete domain of in-line amplitude where lock-in prevails (e.g., 0.190.39 , for $\mathrm{Re}=160$ ) in order to represent a curve.

Abrupt alteration of amplitude This is illustrated in Fig. 9 and can also be expressed in mathematical terms as:

if $0 \leq t<t_{1}$ then $A_{x}=A_{x 1}$; if $t_{1} \leq t \leq t_{F}$ then $A_{x}=A_{x 2}$.

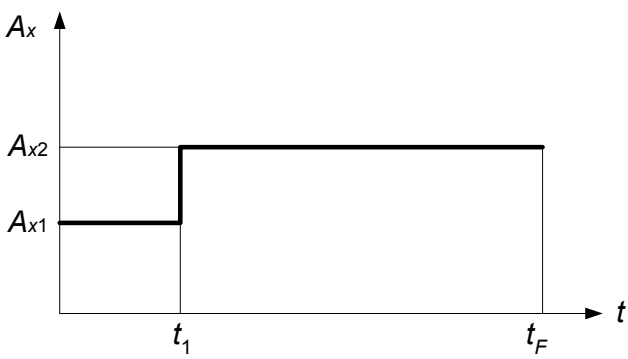

Figure 9. Layout of abrupt amplitude alteration 
An example for the time history of lift is shown in Fig. 10. The amplitude of in-line oscillation is $A_{x}=A_{x 1}=0.25$ until time $t_{1}$, when the amplitude is abruptly changed to $A_{x 2}=0.36$. This value is kept constant during the rest of the computation. While there is some transition, the solution becomes periodic soon after the alteration point $t_{1}$.

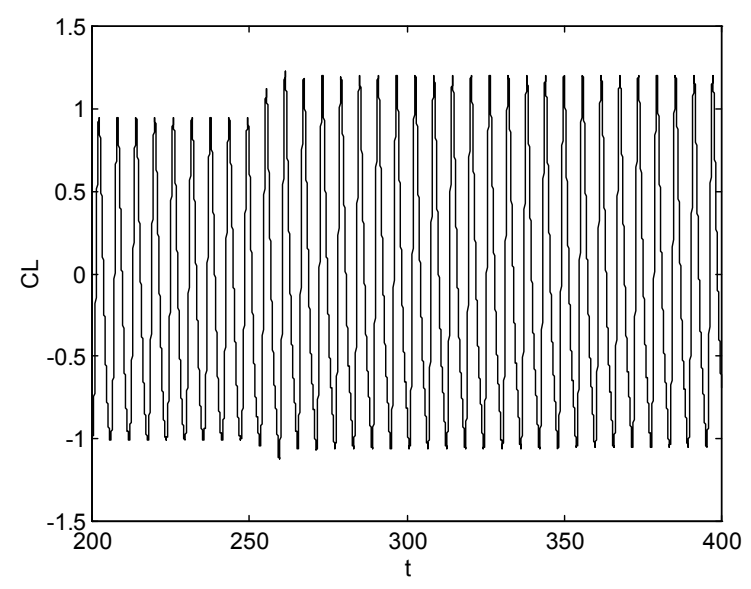

Figure 10. Time history of lift with abrupt amplitude alteration $\left(\operatorname{Re}=160, f=0.9 \mathrm{St}_{0}=0.16938\right)$

The effect of in-line amplitude alteration is demonstrated in Figs. 11-13. Here, Fig. 11 shows the constant-amplitude solution (denoted by empty squares) compared with that when the amplitude is abruptly altered from $A_{x 1}=0.25\left(\Theta=0^{\circ}\right.$ in both cases). This value has been chosen to be directly before a jump in the CA solution (see also Fig. 8). Note that the solution at $A_{x 1}=0.25$ lies on the lower state curve. When the amplitude is then suddenly altered to a different value, the altered-amplitude solution remains on the lower curve, rather than switching, as it does for the constant-amplitude case.

In Fig. 12, however, $A_{x 1}$ was chosen to be 0.251 , which is just after the jump to the upper state curve. When the oscillation is abruptly altered from this value, all points belonging to the altered-amplitude solution remain in the same state curve, i.e., the upper curve. A completely different solution was obtained by using an $A_{x 1}$ value that differed only slightly. This indicates that altering the amplitude of oscillation in the course of computation stabilizes the solution on one state curve, thus avoiding the jumping between states that is a feature of constant-amplitude solutions.

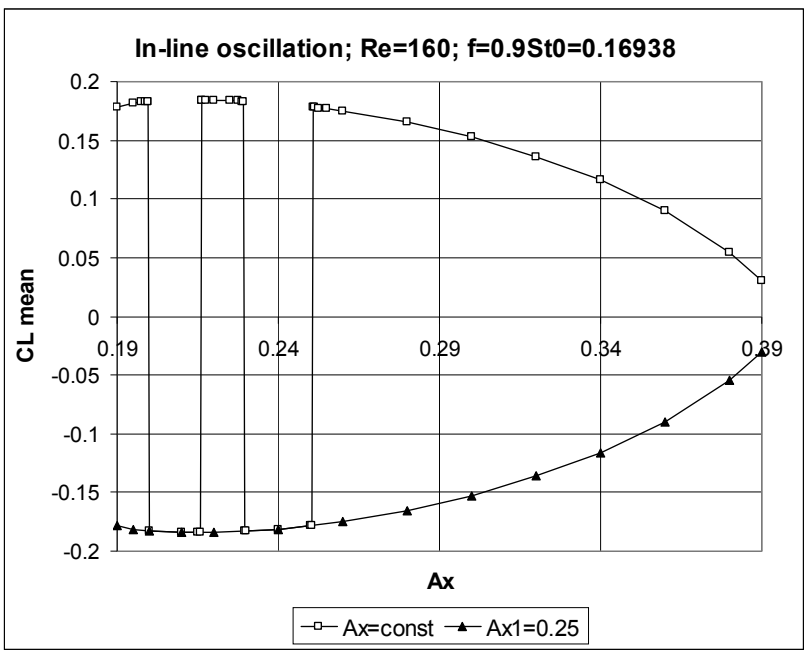

Figure 11. Constant and altered-amplitude solutions for time mean value of lift, $A_{x 1}=0.25$

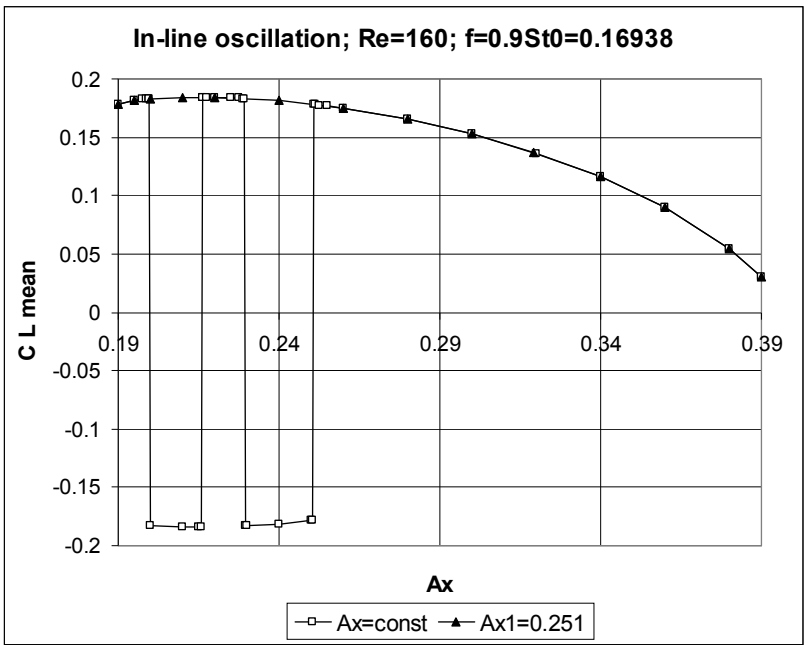

Figure 12. Constant and altered-amplitude solutions for time-mean value of lift, $A_{x 1}=0.251$

Figure 13 represents a case in which the CA solution exhibits only one curve, the results for the altered-amplitude solutions for both $A_{x 1}$ values collapse on the same curve as the CA solution. This was found for all rms curves and the TM of drag and base pressure. 


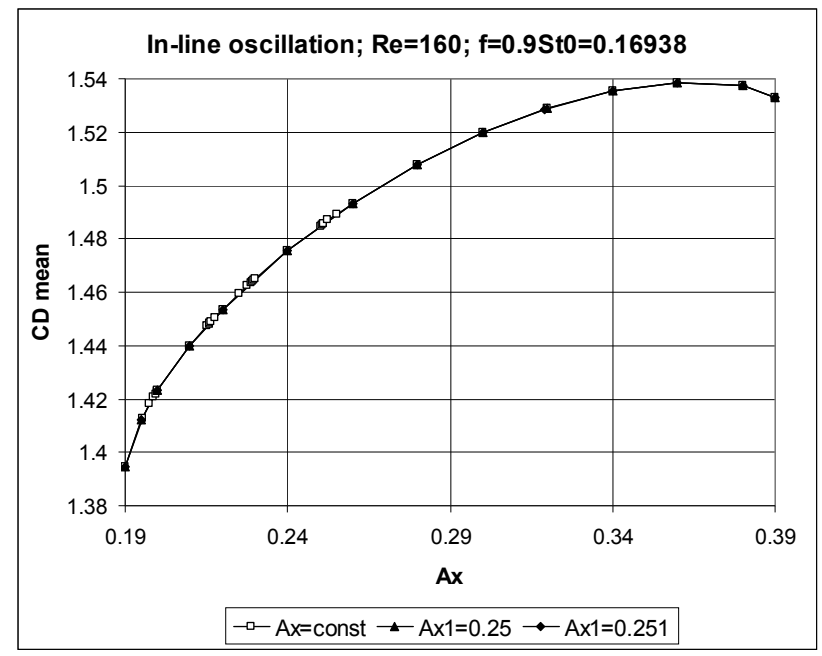

Figure 13. Constant and altered-amplitude solutions for time mean of drag, $A_{x 1}=0.25$ and $A_{x 1}=0.251$

Figure 14 provides some evidence to support the fact that the altered amplitude solution lies on the state curves found in the CA solution. In Fig. 14, three $\left(C_{D}, C_{L}\right)$ limit cycle curves are plotted for $\mathrm{Re}=160, f=0.9 \mathrm{St} 0=0.16938$, at $A_{x}=A_{x 2}=0.26$. The thick line shows the CA solution for $\Theta=0^{\circ}$, corresponding to one point on the upper state curve (see Fig. 11). The lower state curve can be reached at this point by, for example, setting $\Theta$ at $135^{\circ}$ (see Fig. 8), and the corresponding limit cycle is shown by the thin line in Fig. 14. When the altered-amplitude solution started from $A_{x 1}=0.25$ at $\Theta=0^{\circ}$ is plotted, it was found to coincide exactly with the limit cycle curve for the CA solution at $\Theta=135^{\circ}$. This proves that the altered-amplitude solution is indeed on a state curve. These limit cycle investigations were repeated for several points, yielding the same results.

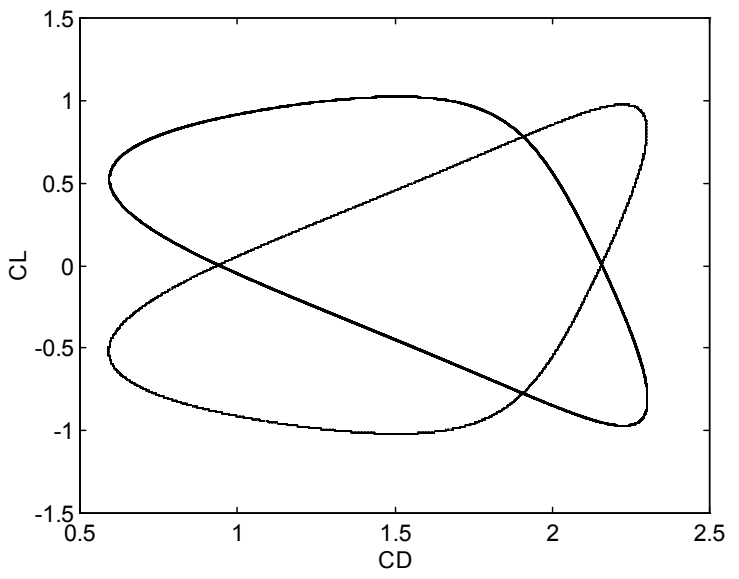

Figure 14. Three $\left(C_{D}, C_{L}\right)$ limit cycle curves at $A_{x}=A_{x 2}=0.26$. Thick line: constant-amplitude (CA) at $\Theta=0^{\circ}$, two coinciding thin lines: $C A$ at $\Theta=135^{\circ}$ and altered amplitude from $A_{x 1}=0.25$ at $\Theta=0^{\circ}$
Figures 11 and 12 suggest that the full state curves can be reproduced not only by using $\mathrm{CA}$ solutions belonging to different initial conditions but also by using an alteredamplitude solution.

Gradual alteration of amplitude It was found that abrupt alteration of the amplitude of oscillation resulted in the altered-amplitude solution remaining in the same state curve. The effect of a gradual alteration in $A_{x}$ was then investigated using the layout shown in Fig. 15.

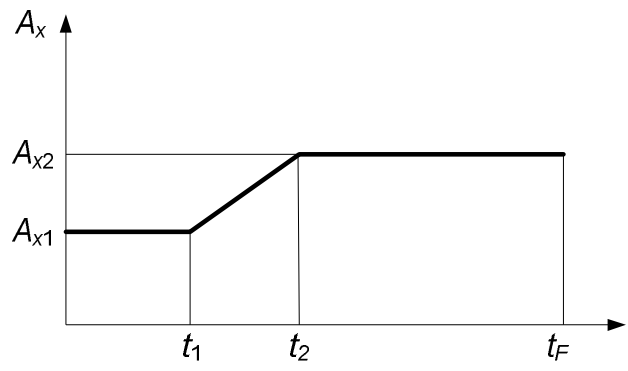

Figure 15. Layout of gradual amplitude alteration

The time history of lift is given in Fig. 16. Here, $\mathrm{Re}=140$, $\Theta=0^{\circ}$, and $f=0.9 \mathrm{St} 0=0.16389$. The amplitude at $t_{1}=250$, $A_{x 1}=0.24$ is changed linearly with time, reaching the value of $A_{x 2}=0.44$ at $t_{2}=450$, and is kept at this value until the final nondimensional time $t_{F}=1000$. Past $t_{2}$, after a short transition, the solution becomes periodic.

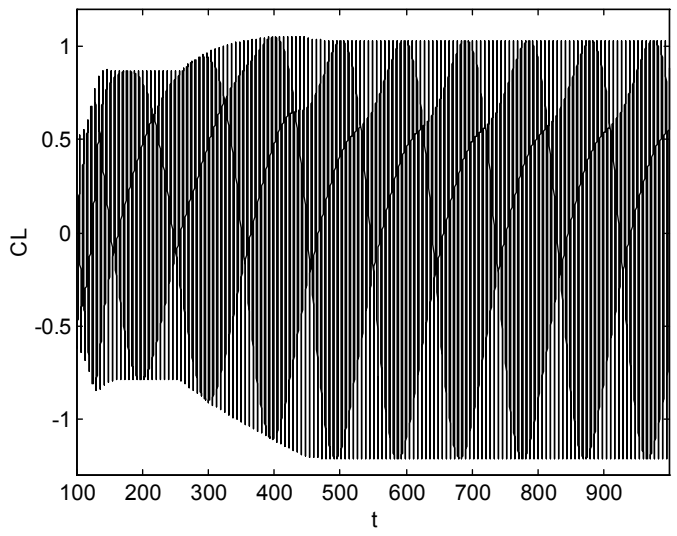

Figure 16. Time history of lift with gradual amplitude alteration $\left(\mathrm{Re}=140, f=0.9 \mathrm{St}_{0}=0.16389\right)$

For purposes of comparison, six different rates of change in amplitude were investigated, ranging from 0.001 to infinity (abrupt alteration), as shown in Table 1. The constant amplitude solution for $A_{x}=0.44$ was also computed. The time history of lift curves for the seven cases investigated are plotted in Fig. 17, between $t=900$ and 1000 ; the curves coincide perfectly, thus appearing as if they were one curve. 
Table 1. Main features of in-line amplitude alteration for seven investigated cases

\begin{tabular}{|l|l|l|l|l|l|}
\hline & $A_{x 1}$ & $A_{x 2}$ & $t_{1}$ & $t_{2}$ & $10 \mathrm{~d} A_{x} / \mathrm{d} t$ \\
\hline Constant \\
amplitude
\end{tabular}

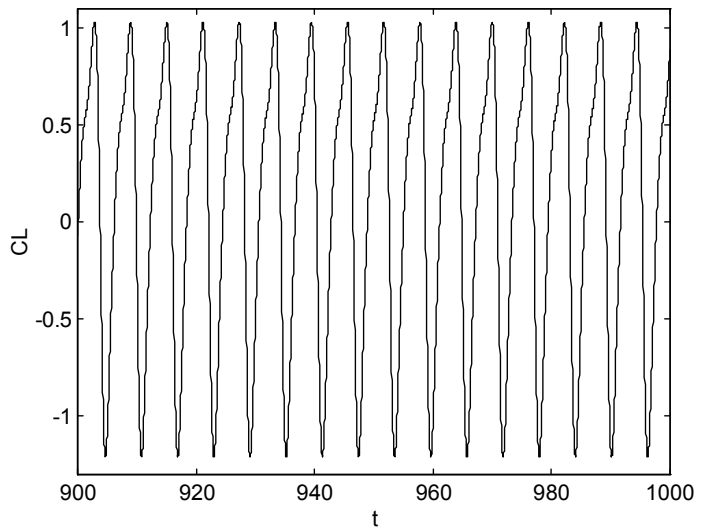

Figure 17. Seven coinciding time history curves of lift

The time history of drag also collapsed into a single curve for the seven cases mentioned, and thus it is no surprise that limit cycles $\left(C_{D}, C_{L}\right)$ for the seven cases mentioned also collapsed on a single curve, as shown in Fig. 18. In conclusion, we can state that the rate of change in the amplitude has no effect on the converged periodic solution: the very slow and the abrupt amplitude alterations yielded the same results.

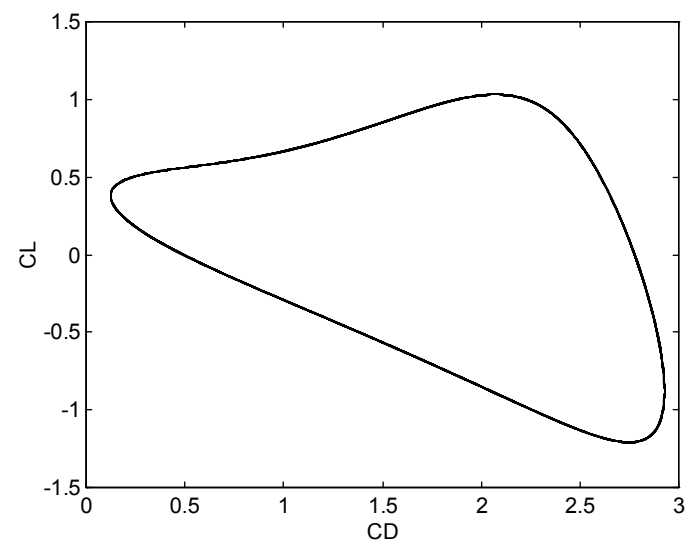

Figure 18. Seven coinciding $\left(C_{D}, C_{L}\right)$ limit cycle curves

\section{TRANSVERSE CYLINDER OSCILLATION}

Constant and altered amplitude solutions were also investigated when the cylinder was oscillated transversely to the main stream. The non-dimensional cylinder displacement of the center of the cylinder is described by

$$
y_{0}=-A_{y} \sin (2 \pi f t+\Theta)
$$

This transverse oscillation can be considered as the limiting case of an orbiting cylinder when the amplitude of in-line oscillation $A_{x}$ tends to zero. For an orbiting cylinder, when the $\mathrm{TM}$ and rms values of force coefficients $\left(C_{L}, C_{D}, C_{p b}, t_{q}\right)$ are plotted against $A_{x}$ while $A_{y}$ is kept constant, no jumps were found in the vicinity of $A_{x}=0$ in any of the eight curves. An example is shown in Fig. 19. The findings for orbital motion are reflected in the results for transverse oscillation, as no jumps are found in any of the curves.

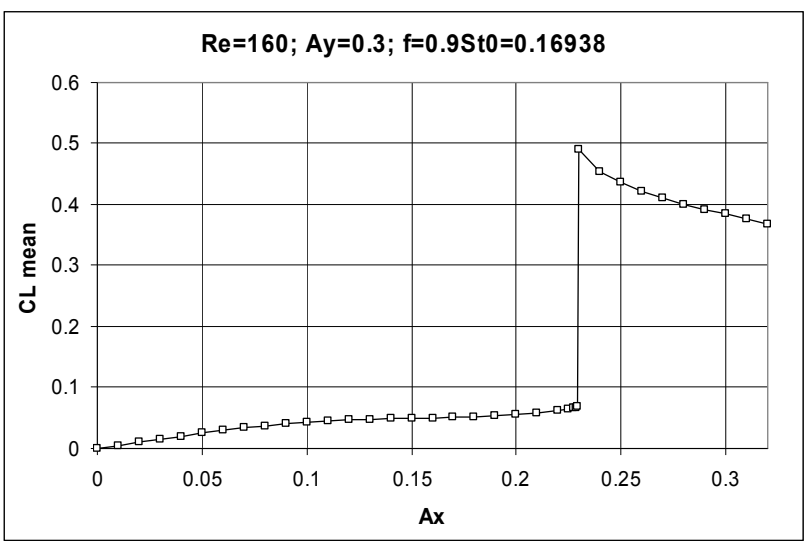

\section{Figure 19. Orbital cylinder motion: TM of lift versus in-line oscillation amplitude}

Another important finding for the transversely oscillating cylinder is that the TM value of lift and torque are zero for all $A_{y}$ oscillation amplitude values investigated. This was also found to be true at $A_{x}=0$ for the limiting case of an orbiting cylinder, as can be seen from Fig. 19 for lift. It seems that although instantaneously the symmetry is broken by the oscillation, over a vortex shedding period there is symmetry.

Systematic computations were carried out for the transversely oscillating cylinder at constant-amplitude (CA) $\left(\mathrm{Re}=160, f=0.9 \mathrm{St} 0=0.16938, \Theta=0^{\circ}\right.$ ) over the amplitude domain where lock-in condition prevailed. When the TM and rms values of force coefficients were plotted against transverse amplitude $A_{y}$, no jumps were detected in any of the coefficients. Similar results were obtained when the effect of frequency ratio on the force coefficients was investigated [23].

The abrupt alteration of transverse amplitude during the course of computation is performed in the same way as for inline oscillation (see Fig. 9). The amplitude of oscillation is $A_{y 1}$ when $0 \leq t<t_{1}$ and at time $t_{1}$ the amplitude is altered abruptly to 
either a smaller or larger value of $A_{y 2}$ and kept at that value for the rest of the computations. The solution is evaluated in the periodic solution domain. $A_{y 2}$ is chosen to cover the complete domain of in-line amplitude where lock-in prevails (e.g., 0.080.45 , for $\mathrm{Re}=160$ ) in order to represent a curve.

As representatives of this type of solutions only two curves are shown here. Figures 20 and 21 show the rms values of lift and torque, respectively. In both figures not only the constantamplitude solutions (empty squares) but the altered amplitude solutions are also shown (filled triangles) for the same initial conditions of $\Theta=0^{\circ}$. As can be seen in these figures, the CA and altered-amplitude solutions coincide and naturally there are no jumps in these curves. Figure 20 also shows the rms value of the "fixed body" lift coefficient for the CA solution (Ay=cont_fb; empty diamonds). It can be seen in Fig. 20 that the rms value of $C_{L f b}$ increases with amplitude $A_{y}$, in contrast with $C_{L r m s}$, which contains the inertial force as well. The variation of $C_{L f b r m s}$ with amplitude $A_{y}$ shows the same trend as that found in [4] for $\mathrm{Re}=400$.

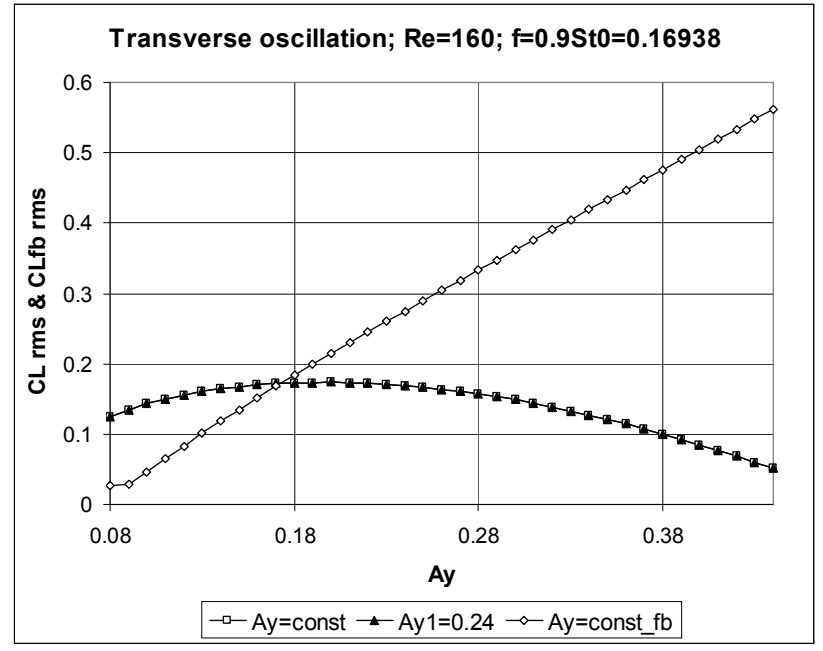

Figure 20. Constant and altered-amplitude solutions for rms of lift, $A_{y 1}=0.24$

Some studies have found switches in vortex structure for transversely oscillating cylinders, but usually above $f / \mathrm{St}_{0}=1$, for $\mathrm{Re}=185$ and a non-dimensional amplitude of 0.2 (e.g., [5], [6], [7]). While [3] reported switches under this value, between $f / \mathrm{St}_{0}=0.875$ and 0.975 , it was at a higher Reynolds number $(\mathrm{Re}=500)$. Our results support these earlier findings.

Since the CA and abruptly-altered solutions agreed perfectly, we did not carry out any investigation of gradual alteration in the amplitude of the transversely oscillated cylinder.

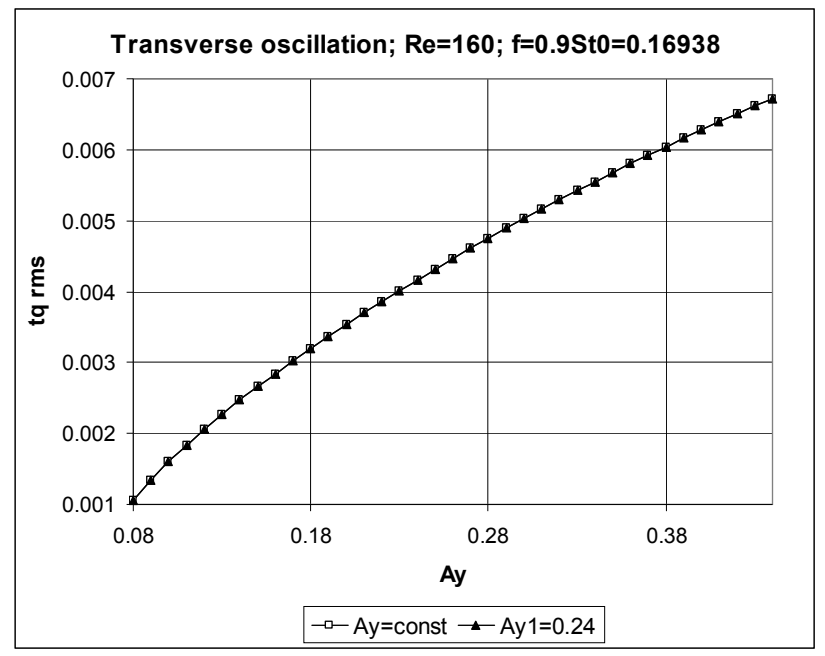

Figure 21. Constant and altered-amplitude solutions for rms of torque, $A_{y 1}=0.24$

\section{CONCLUSIONS}

The effect of oscillation amplitude on the time-mean and rms values of force coefficients when plotted against amplitude was investigated for a cylinder oscillating either in transverse or in-line direction under lock-in conditions.

For in-line oscillation, jumps were found in the TM values of lift and torque coefficients for a constant-amplitude solution. No jumps were detected for the TM of drag or base pressure, nor for the rms of any of the four coefficients. For the TM of lift and torque, when oscillation amplitude was altered abruptly the solution ceased to jump to the other state curve (i.e., ceased to switch vortex structure). Gradual alteration, regardless of the rate of alteration, yielded the same results as those for abrupt alteration: the solution remained on the same state curve thereafter. Since lift in in-line oscillation has a double solution, it would seem necessary for designers to take both solutions for lift force into account when considering transverse load. Amplitude alteration may have potential as a means of limiting the solution to one state curve, although it is difficult to predict which solution would be maintained.

For transverse oscillation, no jumps were found in any TM or rms curves for any of the constant-amplitude solutions. Abrupt alteration had no effect, and gradual alteration was therefore not investigated. Interestingly, the TM value of lift and of torque was zero for all amplitudes investigated.

Although one would expect that the symmetry would be broken for the transversely oscillating cylinder, still it seems that symmetry is maintained over a cycle. Rather surprisingly, it is the in-line oscillation case in which the sudden switches in vortex structure occur. This requires further investigation.

In this study, the investigation was limited to two Reynolds numbers $(\mathrm{Re}=140$ and 160$)$ and one frequency ratio $\left(f / \mathrm{St}_{0}=0.9\right)$. Investigations over a wider parameter range would be 
beneficial. Computations are also planned for the lock-in domain at double the natural vortex shedding frequency $f_{v 0}$ for a cylinder oscillating in-line.

\section{ACKNOWLEDGMENTS}

The support provided by the Hungarian Research Foundation (OTKA, Project No. K 76085) is gratefully acknowledged.

\section{REFERENCES}

[1] Williamson, C. H. K., and Roshko, A., 1988, "Vortex formation in the wake of an oscillating cylinder," Journal of Fluids and Structures 2, pp. 355-381.

[2] Ponta, F.L., and Aref, H., 2006, "Numerical experiments on vortex shedding from an oscillating cylinder," Journal of Fluids and Structures 22, pp. 327-344.

[3] Blackburn, H.M., and Henderson. R.D., 1999, "A study of two-dimensional flow past an oscillating cylinder," Journal of Fluid Mechanics 385, pp. 255-286.

[4] Kaiktsis, L., Triantafyllou, G.S., and Özbas, M., 2007, "Excitation, inertia, and drag forces on a cylinder vibrating transversely to a steady flow," Journal of Fluids and Structures 23, pp. 1-21.

[5] Gu, W., Chyu, C., and Rockwell, D., 1994, "Timing of vortex formation from an oscillating cylinder," Physics of Fluids 6(11), pp. 3677-3682.

[6] Lu, X.Y., and Dalton, C., 1996, "Calculation of the timing of vortex formation from an oscillating cylinder," Journal of Fluids and Structures 10, pp. 527-541.

[7] Guilmineau, E., and Queutey P., 2002, "A numerical simulaton of vortex shedding from an oscillating circular cylinder," Journal of Fluids and Structures 16, pp. 773794.

[8] Leontini, J. S., Stewart, B.E., Thompson, M.C., and Hourigan, K, 2006, "Wake state and energy transitions of an oscillating cylinder at low Reynolds number," Physics of Fluids 18(6), pp. 067101-1-067101-9.

[9] Cetiner, O., and Rockwell, D., 2001, "Streamwise oscillations of a cylinder in a steady current. Part 1. Locked-on states of vortex formation and loading," Journal of Fluid Mechanics 427, pp. 1-28.

[10] Al-Mdallal, Q.M., Lawrence, K.P., and Kocabiyik, S., 2007, "Forced streamwise oscillations of a circular cylinder: Locked-on modes and resulting fluid forces," Journal of Fluids and Structures 23, pp. 681-701.

[11] Rodrigez, M., and Mureithi, N.W., 2006, "Cylinder wake dynamics in the presence of stream-wise harmonic forcing," Proceedings of PVP2006-ICPVT-11, Vancouver, Canada, on CD ROM, pp. 1-9.

[12] Baranyi, L., 2008, "Effect on wake flow of abrupt alterations in ellipticity of an orbiting cylinder," Proc. $9^{\text {th }}$ International Conference on Flow-Induced Vibrations, Prague, pp. 285-290.
[13] Kawamura, T., and Kuwahara, K., 1984. "Computation of high Reynolds number flow around a circular cylinder with surface roughness," Proc. $22^{\text {nd }}$ Aerospace Sci. Meeting, Reno, Nevada, AIAA-84-0340, pp. 1-11.

[14] Baranyi, L., 2003, "Computation of unsteady momentum and heat transfer from a fixed circular cylinder in laminar flow," Journal of Computational and Applied Mechanics 4(1), pp. 13-25.

[15] Baranyi, L., 2008, "Numerical simulation of flow around an orbiting cylinder at different ellipticity values," Journal of Fluids and Structures 24, pp. 883-906.

[16] Kravchenko, A.G., Moin, P., and Shariff, K., 1999. "BSpline method and zonal grids for simulations of complex turbulent flows," Journal of Computational Physics 151, pp. 757-789.

[17] Chakraborty, J., Verma, N., and Chhabra, R.P., 2004. "Wall effects in flow past a circular cylinder in a plane channel: a numerical study," Chemical Engineering and Processing 43, pp. 1529-1537.

[18] Didier, E., and Borges, A.R.J., 2007, "Numerical predictions of low Reynolds number flow over an oscillating circular cylinder," Journal of Computational and Applied Mechanics 8(1), pp. 39-55.

[19] Baranyi, L., 2005, "Lift and drag evaluation in translating and rotating non-inertial systems," Journal of Fluids and Structures 20(1), pp. 25-34.

[20] Bearman, P.W., Graham, J.M.R., Lin, X.W., and Meneghini, J.R., 1995, "Numerical simulation of flowinduced vibration of a circular cylinder in uniform and oscillatory flow," Flow-induced vibration: Proc. 6th International Conference on Flow-Induced Vibration, London, pp. 231-240.

[21] Baranyi, L., 2004, "Numerical simulation of flow past a cylinder in orbital motion," Journal of Computational and Applied Mechanics 5(2), pp. 209-222.

[22] Baranyi, L., and Lewis, R.I., 2006, "Comparison of a gridbased CFD method and vortex dynamics predictions of low Reynolds number cylinder flows," The Aeronautical Journal 110(1103), pp. 63-71.

[23] Baranyi, L., 2008, "Effect of frequency ratio on the force coefficients of a cylinder oscillated in a uniform stream," Proc. 7th JSME-KSME Thermal and Fluids Engineering Conference, Sapporo, Japan, on CD ROM, paper L132, pp.1-4. 